\section{Acute Partial Oculomotor Nerve Palsy and Optic Neuritis Preceding Juvenile Idiopathic Arthritis: A Case Report}

\section{Dear Editor,}

Juvenile idiopathic arthritis (JIA) is the most common pediatric rheumatologic disorder [1]. Although ocular involvement of JIA manifests mostly as uveitis, JIA can also be associated with orbital inflammation like dacryoadenitis and myositis [2,3]. Arthritis usually precedes its uveitis, whereas JIA presenting as uveitis is rarely seen [1]. However, only one case of orbital inflammation has been reported to be an antecedent to JIA [3]. We report a novel case of a child with acute ischemic oculomotor nerve palsy and subsequent optic neuritis preceded the development of systemic-onset form of JIA.

A 30-month-old girl presented with a 2-day history of a painless restricted ocular motility in the right eye 3 days after developing gastroenteritis with high fever, which was found first by her mother. On examination, she fixed and followed well with each eye while she was unable to elevate her right eye above the midline. She showed a right exotropia and right hypotropia without ptosis (Fig. 1A). Both pupils were equal with normal light response. Examination of the left eye was unremarkable. A magnetic resonance imaging (MRI) of the brain and orbits revealed hyper-intense lesions in the right and midline portion of the midbrain around the oculomotor nucleus, consistent with acute infarctions, while the extraocular muscles were normal (Fig. 1B). She had been treated with intravenous ceftriaxone and acyclovir given high-grade persistent fever, erythematous rash, and leukocytosis with lymphocyte dominancy. Despite no signs of other cranial nerve or meningeal involvement and unremarkable cerebrospinal fluid study, she was started on a 5-day course of intravenous dexamethasone and oral aspirin given the abnormal

Received: June 1, 2021 Final revision: July 16, 2021

Accepted: July 28, 2021
MRI findings. Examination of the other major systems including echocardiogram and abdominal ultrasound was normal. The laboratory investigations revealed an increased C-reactive protein $(4.2 \mathrm{mg} / \mathrm{dL}$; normal, $<0.8 \mathrm{mg}$ / $\mathrm{dL}$ ), and anti-cardiolipin IgM (13.5 MPL). Rheumatoid factor, anti-nuclear antibody, and anti-GQ1b antibody were negative. She showed an intermittent exotropia of 20 prism diopters with full duction in the right eye 2 months after tapering oral prednisolone. Two years after oculomotor nerve palsy, at the age of 4 years, she developed a swinging pyrexia and erythematous skin rashes over the trunk for 1 month. Given the uncontrolled high fever and enlarged cervical lymph nodes (LN), the patient underwent LN biopsy showing para-cortical hyperplasia with a negative Epstein-Barr virus in situ hybridization, consistent with chronic inflammation. She reported an abrupt deterioration in the left eye vision, from 20 / 30 to hand movement a day after LN biopsy. Pupil reaction was sluggish in the left eye with a relative afferent pupillary defect. Fundus examination demonstrated mild swelling of the left optic disc (Fig. 1C). A MRI of the brain and orbits revealed swelling and contrast enhancement in the left optic nerve, suggesting optic neuritis and no abnormalities of the brain (Fig. 1D). Anti-myelin oligodendrocyte glycoprotein antibody was negative. The visual acuity in the left eye was restored with resolution of the optic disc swelling on oral prednisolone 3 weeks after a 3-day course of intravenous methylprednisolone. Her pyrexia was also well responded to steroids. One month after the onset of optic neuritis, she had pain in both knees and left wrist with a swollen left knee and a swollen flexed left elbow. Her swinging pyrexia and erythematous rash also returned while oral prednisolone tapering. Elevation of C-reactive protein (up to $7.1 \mathrm{mg}$ / $\mathrm{dL}$ ), interleukin $6, \mathrm{C} 3$, and $\mathrm{C} 4$ was noted. Rheumatoid factor and anti-nuclear antibody were normal. A diagnosis of systemic-onset JIA was made. She was transferred to another hospital for specific management to JIA. Most recently, 8 months after a diagnosis of JIA, visual acuity was $20 / 25$ in the left eye with mild temporal pallor of the optic disc and the rest of ocular finding was unremarkable. Her treatment regimen included naproxen, prednisolone, and methotrexate.

Systemic-onset JIA, previously known as Still's disease, 

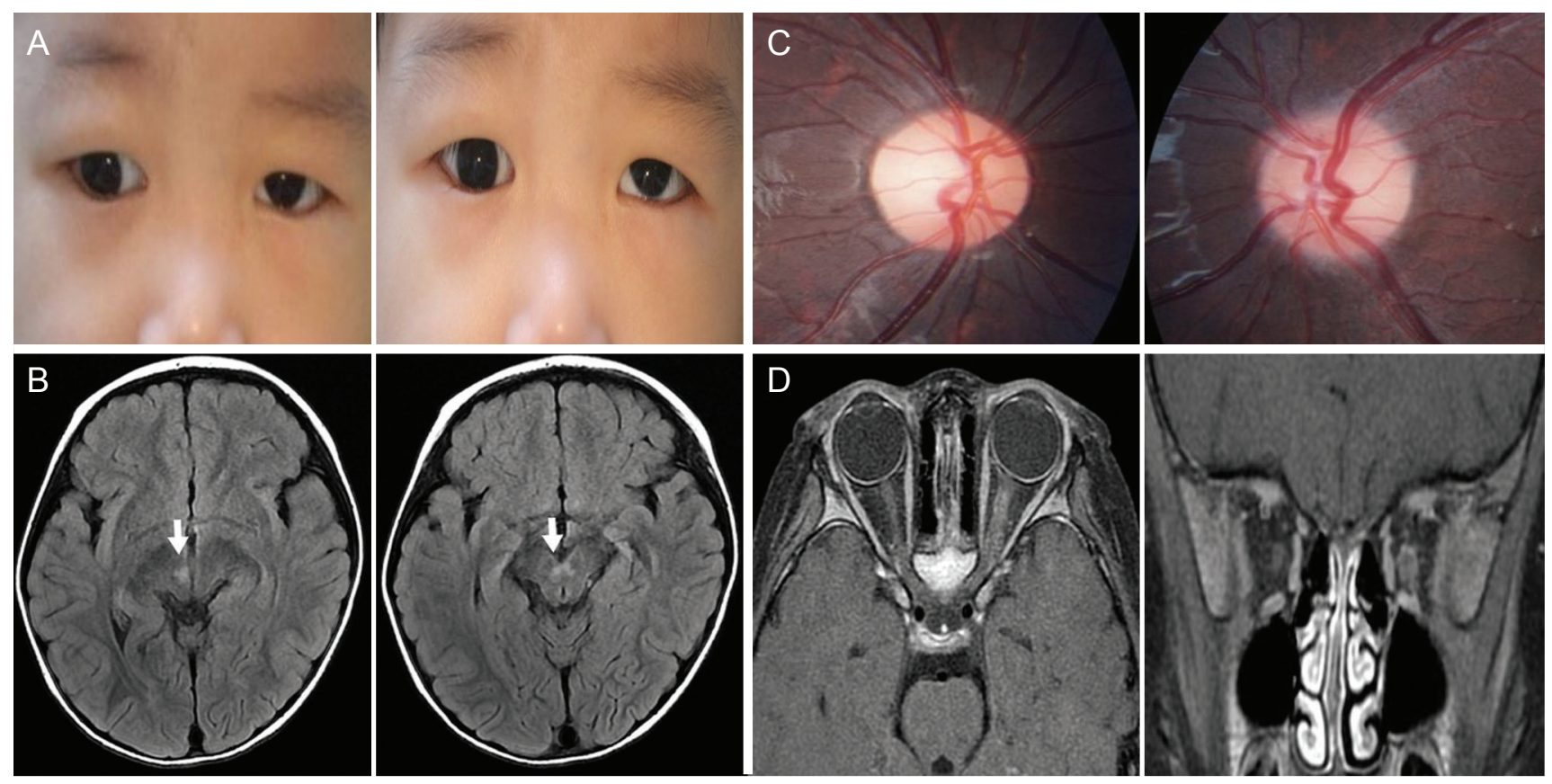

Fig. 1. Gaze photographs and brain magnetic resonance imaging (MRI) when the patient had a right oculomotor nerve palsy at the age of 2 years (A,B) and disc photographs and orbit MRI when she had a left optic neuritis and cervical lymphadenopathy at the age of 4 years (C,D). (A) Gaze photographs show the right exotropia and hypotropia and marked limitation on supra-duction in the right eye. (B) Axial images of gadolinium-enhancing T2-weighted FLAIR brain MRI show hyper-intense lesions in the right and midline portion of the midbrain around third cranial nucleus (white arrows), consistent with acute infarctions. (C) Disc photographs demonstrate mild blurring at the nasal margin of the left optic disc. (D) Axial and coronal views of gadolinium-enhancing T1-weighted fat suppression orbit MRI demonstrate swelling and contrast enhancement of the left optic nerve, suggesting a left optic neuritis. Informed consent for publication of the clinical images was obtained from the parents of patient.

is a minor subtype of JIA with rare eye involvement, which is characterized by arthritis and swinging pyrexia, erythematous rash hepatomegaly or splenomegaly, serositis, and lymphadenopathy [1]. Oculomotor nerve palsy in Kawasaki disease and multiple sclerosis-associated optic neuritis in JIA have been reported [4,5]. However, this is the first report of systemic-onset JIA after ischemic oculomotor palsy and optic neuritis, suggesting its immunologic predisposition.

\section{Seung Ah Chung, Sujung Lee, Seung Woo Kim \\ Department of Ophthalmology, Ajou University School of Medicine, Suwon, Korea \\ E-mail (Seung Ah Chung): sachung@aumc.ac.kr}

\section{Conflict of Interest}

No potential conflict of interest relevant to this article was reported.

\section{References}

1. Yasumura J, Yashiro M, Okamoto N, et al. Clinical features and characteristics of uveitis associated with juvenile idiopathic arthritis in Japan: first report of the pediatric rheumatology association of Japan (PRAJ). Pediatr Rheumatol Online J 2019;17:15.

2. Idowu OO, Saifee M, Copperman TS, Kersten RC. Dacryoadenitis associated with juvenile idiopathic arthritis. Ophthalmic Plast Reconstr Surg 2020;36:e63-5.

3. Tsukikawa M, Lally SE, Shields CL, et al. Idiopathic orbital pseudotumor preceding systemic inflammatory disease in children. J Pediatr Ophthalmol Strabismus 2019;56:373-7.

4. Thapa R, Mallick D, Biswas B, Chakrabartty S. Transient unilateral oculomotor palsy and severe headache in childhood Kawasaki disease. Rheumatol Int 2011;31:97-9.

5. Coskun AN, Gunbey C, Gocmen R, et al. Concurrence of juvenile idiopathic arthritis and primary demyelinating disease in a young child. Mult Scler Relat Disord 2019;27:20-2. 